\title{
A CARB-like $\beta$-lactamase gene from a multiple-drug-resistant Pseudomonas aeruginosa clinical isolate in China
}

Carbenicillin was introduced in therapeutics in 1967 and was found to be particularly useful, either on its own or when associated with aminoglycosides, for the treatment of infections caused by Pseudomonas aeruginosa (Rolinson, 1998), a well-recognized nosocomial pathogen with intrinsic resistance to various antimicrobial agents and the ability to develop multidrug resistance. In 1969, a $\beta$-lactamase was found in $P$. aeruginosa Dalgleish. It was found to be 'markedly active against carbenicillin' and was named PSE-4 (Furth, 1975). Among the carbenicillin-inactivating $\beta$-lactamases, the CARB family is the main group and includes PSE-4 (CARB-1), PSE-1 (CARB-2), CARB-3, CARB-4, CARB-6,

CARB-7, CARB-9 and N29, a $\beta$-lactamase from Proteus mirabilis. In addition, a new group of carbenicillinases, the RTG group, has been proposed, which includes RTG-1 ( $P$. mirabilis GN79 enzyme), RTG-2

(CARB-5) and RTG-3. Although these three chromosomal enzymes show a low level of relatedness to members of the CARB family (44\%), they may be considered to be the ancestors of this enzymic group (Petroni et al., 2004). So far, four genotypes of $b l a_{\mathrm{CARB}}\left(b l a_{\mathrm{CARB}-1}, b l a_{\mathrm{CARB}-2}, b l a_{\mathrm{CARB}-3}\right.$ and $\left.b l a_{\text {CARB-4 }}\right)$ have been discovered in $P$. aeruginosa. We report on a

multiple-drug-resistant $P$. aeruginosa strain from China carrying a CARB-like

$\beta$-lactamase gene.

$P$. aeruginosa WX3225 was isolated in March 2005 from the respiratory tract of a 33-year-old patient undergoing encephalic surgery at Wuxi Fifth People's Hospital, China. The isolate was identified by VITEK Gram-negative identification cards (bioMérieux). A strain of $P$. aeruginosa carrying the $b l_{a_{\mathrm{IMP}-1}}$ gene and a strain of $P$. aeruginosa carrying the $b l a_{\mathrm{VIM}-2}$ gene were used as positive controls for the detection of the $b l a_{\mathrm{IMP}}$ and $b l a_{\mathrm{VIM}-2}$ genes, respectively. $P$. aeruginosa ATCC 27853 was used as a negative control and for quality control of the antimicrobial susceptibility test. Initially, the antimicrobial susceptibility profile of $P$. aeruginosa WX3225 was evaluated using a VITEK system (bioMérieux). MICs were subsequently determined by the microdilution method with cation-adjusted Mueller-Hinton broth (Oxoid) according to the recommendations of the National Committee for Clinical Laboratory Standards. The MICs of potent antibiotics were determined for $P$. aeruginosa WX3225, including ticarcillin, amoxycillin/clavulanic acid, ceftazidime, cefotaxime, cefoxitin, aztreonam, imipenem, gentamicin, netilmicin, tobramycin, amikacin, ciprofloxacin and trimethoprim/sulfamethoxazole (Oxoid). A disc diffusion test, with

2-mercaptopropionic acid as a metallo- $\beta$-lactamase (MBL) inhibitor, was used to screen for MBL producers, as described by Arakawa et al. (2000).

Template DNA for PCR was prepared by a rapid alkaline lysis procedure. The oligonucleotide primers for PCR listed in Table 1 were designed based on $\beta$-lactamase sequences in GenBank. PCR conditions were $3 \mathrm{~min}$ at $93^{\circ} \mathrm{C}$, followed by 40 cycles of $1 \mathrm{~min}$ at $93^{\circ} \mathrm{C}, 1 \mathrm{~min}$ at $55^{\circ} \mathrm{C}$ and $1 \mathrm{~min}$ at $72^{\circ} \mathrm{C}$, with a final extension for $7 \mathrm{~min}$ at $72^{\circ} \mathrm{C}$. Amplicons were purified with PCR clean-up kits (Roche Molecular Biochemicals) and sequenced on an ABI PRISM 377 sequencer (Applied Biosystems).

$P$. aeruginosa WX3225 was highly resistant to imipenem and to the other $\beta$-lactams except for aztreonam and production of MBL was demonstrated by screening with the disc diffusion test. P. aeruginosa WX3225 was positive in PCR assays with $b l a_{\mathrm{CARB}^{-}}, b l a_{\mathrm{VIM}^{-}}$and $b l a_{\mathrm{TEM}^{-}}$-specific oligonucleotide primers. The $b l a_{\mathrm{TEM}}$ and $b l a_{\text {VIM }}$ gene sequences were analysed by BLAST and found to be identical to $b l a_{\mathrm{TEM}-1}$ and $b l a_{\mathrm{VIM}-2}$, respectively. Analysis of the $b l a_{\mathrm{CARB}}$ gene sequence revealed $99 \cdot 4 \%$ nucleotide identity with the $b l a_{\mathrm{CARB}-4}$ gene (GenBank accession no. U14749) with replacements of $\mathrm{G} \rightarrow \mathrm{A}, \mathrm{C} \rightarrow \mathrm{A}$ and $\mathrm{T} \rightarrow \mathrm{A}$ at nt 277,471 and 520 of the structural gene, respectively. Besides the synonymous mutation at nt 471, two non-synonymous mutations differentiated $b l a_{\mathrm{CARB}-4}$ from the $P$. aeruginosa WX3225 bla $a_{\mathrm{CARB}}$ gene sequence, resulting in amino acid changes of ${ }^{93} \mathrm{Glu} \rightarrow$ Lys and ${ }^{174} \mathrm{Ser} \rightarrow$ Thr. The $b l a_{\mathrm{CARB}}$ gene was a novel $\beta$-lactamase gene and was tentatively named a $b l a_{\mathrm{CARB}}$-like gene.

To our knowledge, the combination of $b l a_{\mathrm{VIM}-2}$ associated with carbenicillinase has been described previously only in strains from Portugal (Quinteira et al., 2005). However, the combination of both VIM-2 and TEM-1 associated with carbenicillinase, as reported here, has not been described previously. This is the first report of $b l a_{\mathrm{CARB}}$ in China. $\beta$-Lactamase production is a major clinical and public health problem as it has been increasingly reported and it poses a challenge to antimicrobial therapy. Some structural genes for $\beta$-lactamases have been located on resistance cassettes as part of integrons that have been characterized as genetic elements capable of capturing and rearranging resistance genes (Philippon \& Arlet, 2006). Continuous epidemiological surveillance of $\beta$-lactamase production is needed in institutions.

\section{Acknowledgements}

We are grateful to Yun-song Yu, Department of Infectious Diseases, First Affiliated Hospital, College of Medicine, Zhejiang University, Hangzhou, China, for his generous provision of the $b l a_{\mathrm{IMP}^{-}}$and $b l a_{\mathrm{VIM}}$-positive control strains and for technical assistance.

The GenBank/EMBL/DDBJ accession number of the sequence determined in this work is DQ309772.

Jidong Wang, ${ }^{1}$ Ruifang Bo, ${ }^{2}$ Lan $\mathrm{Xu},{ }^{2}$ Zuhuang $\mathrm{Mi}^{3}$ and Chunxin Wang

${ }^{1}$ Wuxi Fifth People's Hospital, Wuxi 214073, China

${ }^{2}$ Wuxi First Hospital Affiliated to Nanjing Medical University, Wuxi 214002, China 
Table 1. Sequences of the $\beta$-lactamase gene primers used in this study

\begin{tabular}{|c|c|c|}
\hline Gene & Primer sequence $\left(5^{\prime} \rightarrow 3^{\prime}\right)$ & Expected PCR product (bp) \\
\hline \multirow[t]{2}{*}{ IMP } & P1: CGGCC(G/T)CAGGAG(A/C)G(G/T)CTTT & 587 \\
\hline & P2: AACCAGTTTTGC(C/T)TTAC(C/T)AT & \\
\hline \multirow[t]{2}{*}{ VIM } & P1: ATTCCGGTCGG(A/G)GAGGTCCG & 633 \\
\hline & P2: GAGCAAGTCTAGACCGCCCG & \\
\hline \multirow[t]{2}{*}{ DHA } & P1: AACTTTCACAGGTGTGCTGGGT & 405 \\
\hline & P2: CCGTACGCATACTGGCTTTGC & \\
\hline \multirow[t]{2}{*}{ GES } & P1: ATGCGCTTCATTCACGCAC & 846 \\
\hline & P2: CTATTTGTCCGTGCTCAGG & \\
\hline \multirow[t]{2}{*}{ CARB } & P1: AAAGCAGATCTTGTGACCTATTC & 588 \\
\hline & P2: TCAGCGCGACTGTGATGTATAAAC & \\
\hline \multirow[t]{2}{*}{ TEM } & P1: AGGAAGAGTATGATTCAACA & 535 \\
\hline & P2: CTCGTCGTTTGGTATGGC & \\
\hline \multirow[t]{2}{*}{ SHV } & P1: GGCTATGCGTTATATTCGCC & 867 \\
\hline & P2: GGTTAGCGTTGCCAGTGC & \\
\hline \multirow[t]{2}{*}{ CTX-M-1 group } & P1: ATGGTTAAAAAATCACTGCGC & 833 \\
\hline & P2: TCCCGACGGCTTTCCGCCTT & \\
\hline \multirow[t]{2}{*}{ OXA-10 group } & P1: GTCTTTC(A/G)AGTACGGCATTA & 822 \\
\hline & P2: GATTTTCTTAGCGGCAACTTA & \\
\hline \multirow[t]{2}{*}{ PER } & P1: AGTCAGCGGCTTAGATA & 978 \\
\hline & P2: CGTATGAAAAGGACAATC & \\
\hline \multirow[t]{2}{*}{ VEB } & P1: GCGGTAATTTAACCAGA & 961 \\
\hline & P2: GCCTATGAGCCAGTGTT & \\
\hline
\end{tabular}

${ }^{3}$ Wuxi Clone Gen-Tech Institute, Wuxi 214026, China

Correspondence: Chunxin Wang (bacteria001@163.com)

Arakawa, Y., Shibata, N., Shibayama, K. Kurokawa, H., Yagi, T., Fujiwara, H. \& Goto, M. (2000). Convenient test for screening metallo- $\beta$ lactamase-producing Gram-negative bacteria by using thiol compounds. J Clin Microbiol 38, $40-43$.
Furth, A. J. (1975). Purification and properties of a constitutive $\beta$-lactamase from Pseudomonas aeruginosa strain Dalgleish. Biochim Biophys Acta 377, 431-443.

Petroni, A., Melano, R. G., Saka, H. A. \& 9 other authors (2004). CARB-9, a carbenicillinase encoded in the VCR region of Vibrio cholerae non-O1, non-O139 belongs to a family of cassette-encoded $\beta$-lactamases. Antimicrob Agents Chemother 48 4042-4046.
Philippon, A. \& Arlet, G. (2006). $\beta$-Lactamases of Gram-negative bacteria: never-ending clockwork! Ann Biol Clin 64, 37-51 (in French).

Quinteira, S., Sousa, J. C. \& Peixe, L. (2005). Characterization of In100, a new integron carrying a metallo- $\beta$-lactamase and a carbenicillinase, from Pseudomonas aeruginosa. Antimicrob Agents Chemother 49, 451-453.

Rolinson, G. N. (1998). Forty years of $\beta$-lactam research. J Antimicrob Chemother 41, 589-603. 